CLINICAL STUDY

\title{
Increased mammographic breast density in acromegaly: quantitative and qualitative assessment
}

\author{
Alberto Tagliafico $^{1}$, Massimo Calabrese ${ }^{1}$, Giulio Tagliafico ${ }^{2}$, Eugenia Resmini $^{3,5}$, Carlo Martinoli $^{4}$, Alberto Rebora ${ }^{5}$, \\ Annamaria Colao $^{6}$, Rosario Pivonello ${ }^{6}$ and Diego Ferone ${ }^{5}$ \\ ${ }^{1}$ Department of Radiology, National Institute for Cancer Research (IST) and ${ }^{2}$ Department of Engineering, University of Genova, Genova 16132, Italy, \\ ${ }^{3}$ Instituto de Investigación Biomédica (IIB)-Sant Pau, Endocrinology/Medicine Department and Centro de Investigación Biomédica en Red de Enfermedades \\ Raras (CIBER-ER, Unidad 747), ISCIII; Universitat Autonoma de Barcelona, Barcelona 08025, Spain, ${ }^{4}$ Department of Radiology, DISC, ${ }^{5}$ Department of \\ Endocrinological and Medical Sciences (DiSEM) and Center of Excellence for Biomedical Research, University of Genova, Genova 16132, Italy and \\ ${ }^{6}$ Department of Molecular and Clinical Endocrinology and Oncology, Federico II University of Naples, Naples 80126, Italy \\ (Correspondence should be addressed to A Tagliafico; Email: atagliafico@sirm.org)
}

\begin{abstract}
Context: Mammographic density is a strong independent risk factor for breast cancer, whose prevalence in acromegaly is still controversial.

Objective: To compare breast density in premenopausal acromegalic patients and controls and to determine whether density correlated with disease duration, GH, and IGF1 levels.

Design, setting and participants: A prospective study involving 30 patients and 60 controls matched for age and body mass index.

Interventions: A quantitative computer-aided mammographic density estimation (MDEST) and a qualitative blind evaluation by two experienced radiologists using the breast imaging reporting and data system (BI-RADS) was performed. Totally, 60 (acromegaly) and 120 (controls) craniocaudal and mediolateral oblique mammograms were evaluated in both patients and controls.

Main outcome measures: Breast density.

Results: Patients showed a significantly $(P<0.01)$ increased mammographic breast density with both methods (MDEST: $0.33 \pm 0.21 \%$ and BI-RADS category: $2.81 \pm 0.78$ ) in comparison with controls (MDEST: $0.26 \pm 0.19 \%$ and BI-RADS category: $2.35 \pm 0.61$ ). The agreement between the two methods and inter-observer agreement between the two radiologists were excellent $(k=0.63$ and $k=0.85)$. In patients grouped according to disease activity (17 controlled and 13 uncontrolled) and medical therapy (15 treated and 15 untreated), no differences were found. All these groups had significantly increased mammographic breast density compared with controls $(P<0.01)$.

A positive correlation was found between mammographic breast density, IGF1 values and disease duration ( $r=0.29$ and $r=0.39)$, whereas it was not found with $\mathrm{GH}(r=-0.02)$.

Conclusions: Mammographic breast density in premenopausal acromegalic patients is significantly higher than controls and positively correlated with IGF1 and disease duration.
\end{abstract}

European Journal of Endocrinology 164 335-340

\section{Introduction}

The clinical importance of mammographic percent density is twofold: first it decreases the sensitivity of mammography in detection of breast carcinoma, because dense fibroglandular tissue may obscure pathological findings; secondly, the risk of developing breast cancer is directly associated with increased mammographic density, and a substantial fraction of breast cancers can be attributed to this risk factor (1). Women with high mammographic density have a fiveto six-fold increased risk of breast cancer $(2,3)$.

Several studies have investigated the relationship between mammographic percent density and hormones; however, the results are unclear so far (4-6).
A positive association between mammographic breast density and plasma insulin-like growth factor 1 (IGF1) in premenopausal women has been observed in three studies (4-6), whereas a fourth one did not confirm this evidence (7). GH is mitogenic and anti-apoptotic in several tissues $(5,6)$, and IGF1 is an important mitogen as well, required by different cell types to progress from the G1 to the S phase of the cell cycle (6). Acromegaly is characterized by elevated GH and IGF1 levels, and although overall cancer incidence in acromegalic patients is not increased, there is a risk of developing colorectal cancer. Uncontrolled GH levels likely provide a growth advantage to neoplasms, resulting in more aggressive disease and increased cancer-associated mortality. More recently, an increased prevalence of 
thyroid cancer has been suggested as well, and an association between tumor growth and GH or IGF1 levels has been alternatively suggested. The data on the prevalence of breast and prostate cancer in acromegaly are still controversial (8).

Further knowledge of the relationship between mammographic density, circulating biomarkers and clinical features related to breast cancer risk may, therefore, elucidate disease mechanisms and offer clues to more specific prevention strategies not only in acromegalic patients but also in the general population (9).

To the best of our knowledge, there are no data in literature investigating mammographic breast density in acromegaly. Therefore, the aim of our study was to investigate breast density in acromegaly in comparison with healthy women, and to determine whether breast density might correlate with disease duration, $\mathrm{GH}$, and IGF1 levels in premenopausal acromegalic women.

\section{Materials and methods}

\section{Patients}

A total of 30 premenopausal acromegalic patients (age: range $22-45$ years; mean \pm s.e.M. $41.8 \pm 1.2$; body mass index (BMI): 24.9 \pm 2.81 ; disease duration: range 2-14 years; mean \pm s.E.M. $4.92 \pm 0.91$ ) have been evaluated between May 2007 and December 2007. On the basis of an interview with the referring clinician, all patients referred to have a spontaneous and regular cycle.

The diagnosis of acromegaly was based on established criteria (10), and gonadotropin (LH and FSH), estrogen levels, and menstrual cycle history were evaluated as well. Disease activity was evaluated by means of the oral glucose tolerance test (OGTT), with a single blood sample being collected at 120th min and the basal value of IGF1 (10). Patients were subdivided into 'controlled' and 'uncontrolled' according to the evaluation of acromegaly activity based on the lack of GH suppression during OGTT, coupled with elevated IGF1 levels and 'partially controlled', when a discordance among GH after OGTT and basal IGF1 values was recorded.

Postmenopausal women (both natural and surgery induced), smokers, patients taking hormone medications (including oral contraceptives within 3 months from the mammography) tamoxifen or raloxifene, pregnant women, patients with a history of cancer at any site, or with breast reduction or implants, and with diabetes mellitus, renal or hepatic disease (11) were excluded.

At the beginning of the study, 8 newly diagnosed patients were untreated, 22 had undergone transsphenoidal surgery (15 also treated with somatostatin analogs).

For a further statistical analysis, women were subdivided into treated $(n=15)$ and untreated $(n=15)$ according to whether they were receiving medical treatment with somatostatin analogs at the beginning of the study. Patients were also subdivided into two subgroups, according to disease activity: controlled $(n=17)$ and uncontrolled $(n=13)$. The control group included 60 healthy volunteers who received a screening mammogram, matched for age and body mass (age: range, 23-47 years; mean \pm s.e.м. 41.9 \pm 4.62 ; BMI: mean \pm s.e.M. 25.1 \pm 0.75$)$. All of them respected the same exclusion criteria adopted for acromegalic women.

The study protocol was approved by the local ethical committee, and a written informed consent was obtained from all patients and controls.

\section{Mammogram selection and digitization}

The dataset, normally used at our institution for the screening program, comprised of four-view craniocaudal (CC) and mediolateral oblique (MLO) mammograms.

The mammograms had been acquired by using Mammography Quality Standards Act (MQSA)approved GE DMR mammography units with Kodak MR2000 (Kodak) screen and film systems. All images were digitized by using a laser film scanner (Lumisys, Mountain View, CA, USA) with a pixel size of $0.05 \times 0.05 \mathrm{~mm}$ and 4096 gray levels. The gray levels were linearly proportional to the optical densities, from 0.1 to $\sim 4.0$ optical density units. The nominal optical density range of the scanner is $0-4$, with large pixel values corresponding to low optical density. In our study, the breast density pattern was analyzed in high spatial resolution (pixel size of $0.005 \mathrm{~mm}$ ); the full-spatial resolution was $2800 \times 2400$ pixels in size. The software used to analyze breast density was developed by an engineer with a 3 year of experience in this field (G T) using a new software for breast density estimation recently developed (12).

\section{Mammogram density analysis with MDEST}

The background features were automatically excluded by the software, while the pectoralis muscle was manually excluded. Breast boundary was automatically calculated by using a gradient-based edge-tracking algorithm. After the creation of a threshold value, pixels were separated in two classes: pixel values above the threshold corresponded to dense tissue, and the class of pixel values below the threshold corresponded to fat tissue (Fig. 1). Breast density was then calculated dividing the number of pixels in the dense area by the total number of pixels in the entire breast region as described elsewhere (12).

\section{Qualitative BI-RADS density classifications}

Mammographic breast density was classified using the American College of Radiology Breast Imaging 


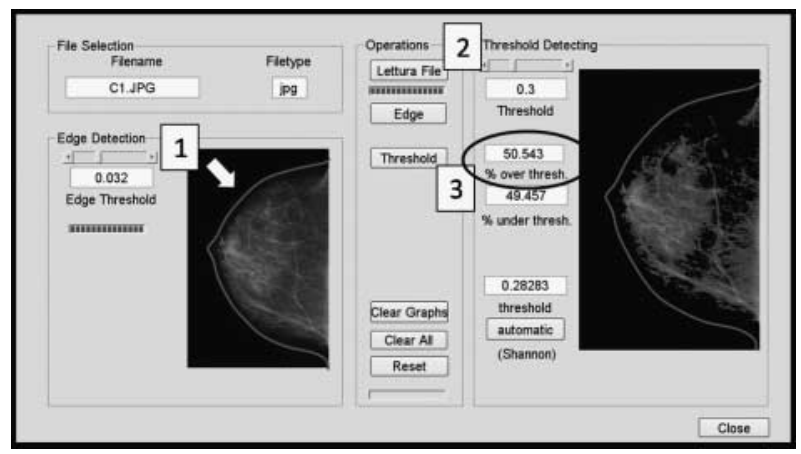

Figure 1 Quantitative assessment of breast density. The figure shows the computer interface. At the first step (1), the edges of the breast are identified, then the radiologist adjusts the density threshold in the semi-automated method (2), and finally the percentage of dense breast is read into the black circle (3). In the fully automated method, there is no need to adjust the threshold (2). On the left, the selection of the breast edge can be visualized (in this figure, it is artificially thickened for visual purposes), while on the right, there is the mask of the breast dense tissue. In this example, the breast is $50 \%$ dense.

Reporting and Data System (BI-RADS) (13) and divided into four morphological categories according to the parenchymal structure (BI-RADS 1: the breast is almost entirely fatty; BI-RADS 2: scattered fibroglandular densities; BI-RADS 3: the breast is heterogeneously dense, and mammographic sensitivity can be reduced; BI-RADS 4: the breast tissue is extremely dense, and lesions may not be detected at mammography).

The mammograms were also divided into four density categories, which corresponded to the parenchymal structure (1: $0-25 \% ; 2: 25-50 \% ; 3: 50-75 \% ; 4>75 \%)$. These procedures were performed by two blinded radiologists ( $\mathrm{M} \mathrm{C}$ and $\mathrm{E} \mathrm{B}$ ) with 20 and 25 years of experience respectively in breast examination. Since elevated mammographic densities (BI-RADS 3 and 4) may reduce mammographic sensitivity, an ultrasonographic examination was immediately performed in the women belonging to these groups by one of the experienced radiologist (M C). Totally, 60 and 120 CC and MLO mammograms were evaluated in both acromegalic patients and controls.

\section{Laboratory assays}

Serum GH levels were determined by means of a chemiluminescent ICMA assay (Immulite; D.P.C., Los Angeles, CA, USA). The analytical sensitivity of this assay was $0.01 \mu \mathrm{g} / \mathrm{l}$, and the accuracy was $<7 \%$ in the standard curve range; the standard curve was calibrated against WHO 1st IRP 80/505 $(1 \mathrm{mg}=2.6 \mathrm{IU})$. All these data have been validated in our laboratory.

IGF1 was measured by RIA using immunochemicals and tracer provided as reported previously (14).

\section{Statistical analysis}

Statistical analysis was performed using the MannWhitney $U$ test for unpaired data to compare patients and healthy controls. Comparison within patients' groups was made using the Mann-Whitney $U$ test. Values were expressed as mean \pm s.D. for the radiological data and mean \pm s.E.M. for the endocrinological data. $P$ values $<0.05$ were considered statistically significant. The Pearson test and linear regression analysis were used to correlate mammographic breast density with other parameters. Multivariate analysis was performed with SPSS software (SPSS Inc., Chicago, IL, USA) to assess whether IGF1 remains positively correlated with breast density after adjustments for BMI and insulin resistance.

\section{Results}

Acromegalic patients showed a significantly $(P<0.01)$ increased mammographic breast density with both methods (mammographic density estimation (MDEST) density (\%): $0.33 \pm 0.21$ and BI-RADS category: 2.81 \pm 0.78 ) in comparison with controls (MDEST density (\%): $0.26 \pm 0.19$ and BI-RADS category: $2.35 \pm 0.61$ ).

An overall $7 \%$ of increased mammographic breast density was observed in acromegalic patients in comparison with controls (Table 1).

The agreement between the two methods and interobserver agreement between the two radiologists were excellent $(k=0.63$ and $k=0.85)$.

Acromegalic patients had significantly increased mammographic breast density in comparison with controls $(P<0.04)$.

Moreover, no differences were found between controlled and uncontrolled or treated and untreated patients. All these groups had significantly increased mammographic breast density compared with controls $(P<0.01)$.

A positive correlation between IGF1 values, disease duration and mammographic breast density $(r=0.29$; $P<0.01$ and $r=0.39: P<0.01$ ) was found (Fig. 2). No correlation with $\mathrm{GH}$ levels was observed $(r=-0.02$;

Table 1 Clinical and mammographic data of the acromegalic patients and controls.

\begin{tabular}{lccc}
\hline & $\begin{array}{c}\text { Acromegaly } \\
(n=30)\end{array}$ & $\begin{array}{c}\text { Control } \\
(n=60)\end{array}$ & $\boldsymbol{P}$ value \\
\hline Age (years) & $41.8 \pm 1.2$ & $41.9 \pm 4.62$ & $<0.54$ \\
Body mass index (BMI) & $24.9 \pm 2.81$ & $25.1 \pm 0.75$ & $<0.78$ \\
Disease duration (years) & $4.92 \pm 0.91$ & & \\
IGF1 SDS & $3.12 \pm 0.35$ & & \\
GH nadir (ng/ml) & $0.98 \pm 0.89$ & & \\
Breast density (MDEST\%) & $0.33 \pm 0.21$ & $0.26 \pm 0.19$ & $<0.01$ \\
Breast density (BI-RADS) & $2.81 \pm 0.78$ & $2.35 \pm 0.61$ & $<0.01$ \\
\hline
\end{tabular}




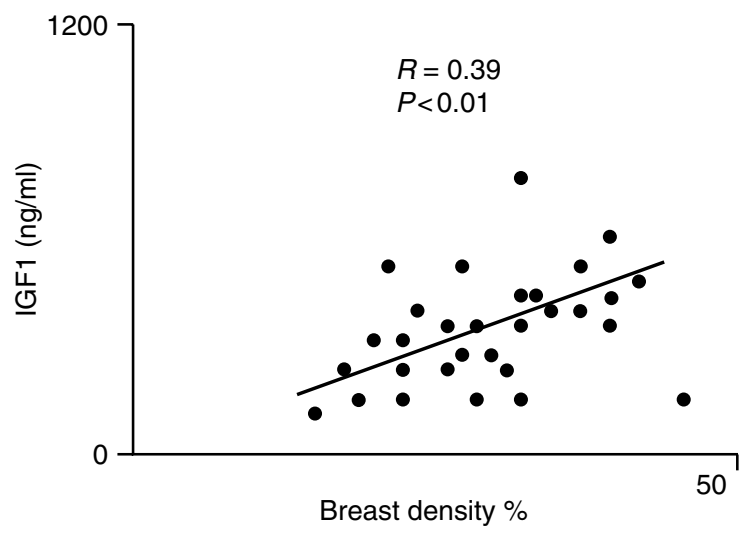

Figure 2 Correlation between IGF1 values and breast density expressed as percentage.

$P<0.01)$. Multivariate analyses confirmed that IGF1 remains correlated with breast density after adjustment for BMI and insulin resistance $(P<0.04$; 95\% confidence intervals: 0.003 and 0.333).

\section{Discussion}

Although still controversial, some evidence suggested that breast malignancies might be increased in acromegaly $(15,16)$.

It has been demonstrated that breast cancer risk rose with an increasing percentage of the breast area $(1,3,4)$. This association was not explained by other risk factors such as age, weight, age at first childbirth, family history of breast cancer, alcohol use, prior benign breast disease, age at menarche, and age at menopause (5). It has also been demonstrated that a positive association between plasma IGF1 concentration and both percent and absolute mammographic densities is present in postmenopausal women who were not using hormonal therapy (17). Given the association between breast density and IGF1 concentrations, the purpose of the study was to assess whether breast density is increased in acromegalic patients and to investigate relationships between breast density, GH and IGF1 values in these patients.

The results demonstrated an overall $7 \%$ of increased mammographic breast density in acromegalic patients in comparison with controls. In line with the recent literature, these data suggest that acromegalic patients might have an increased risk of developing breast cancer compared with the normal population. Considering that the relative risk of breast cancer increases $\sim 2 \%$ by every $1 \%$ increase in breast density (18), a $7 \%$ of increased mammographic breast density in acromegaly may be, in theory, responsible of increased risk more than $10 \%$.
Moreover, our results support a positive association between mammographic density and IGF1 levels and disease duration, suggesting that IGF1, rather than GH, might be responsible for the increased breast density (19). IGF1 is an important mitogen, which exerts powerful effects on each of the key stages of cancer development and behavior: cellular proliferation and apoptosis, angiogenesis and metastasis, and more recently, development of resistance to chemotherapeutic agents (20). It is a potent proliferative agent affecting almost every cell type. In addition to these proliferative actions, IGF1 also acts as an anti-apoptotic agent, influencing the responses to a variety of agents in numerous cell types (20). On the basis of these data, breast cancer risk in acromegalic patients should be increased; however results from epidemiological studies are controversial (20). A recent study confirmed that high levels of GH and IGF1 in premenopausal women increase the risk of breast cancer because $65 \%$ of patients with breast cancer are diagnosed in the premenopausal period, and initial GH and IGF1 levels of all patients with breast cancer are high (21). In a study by Nabarro (22), it was shown that there was a fourfold increase in breast cancer incidence in acromegalic patients (22). Indeed, increased levels of GH and IGF1 may stimulate breast fibroglandular tissue determining an increased breast density, which may be one of the factors determining an increased breast cancer risk in acromegaly $(23,24)$. However, other factors, namely genetic predisposition, peripheral and local activity of IGF-binding proteins, local immune milieu, as well as metabolic status, may influence this picture and probably explain the conflicting results from the epidemiological studies $(25,26)$. The lack of difference between controlled and uncontrolled patients is probably due to the small sample; however, it is unclear whether reaching the statistical significance for controlled and uncontrolled patients may have a clinical significance.

From the radiological point of view, an increased breast density reduces mammographic diagnostic accuracy (12); therefore, in acromegalic patients, screening programs including only mammography may have a reduced cancer detection rate (27). On the light of increased mammographic breast density, acromegalic patients may be evaluated with the combined use of mammography and ultrasonography or may be inserted in magnetic resonance imaging screening programs for high-risk women (28). In interpreting repeated results, for example pre- and post-therapy, different factors have to be considered such as age and BMI, the use of tamoxifen in patients suffering from breast cancer, estrogen-plus-progesterone and estrogen-alone menopausal hormone therapy. These factors may influence breast density and have to be corrected to perform an accurate analysis.

In conclusion, our study demonstrates that mammographic breast density is significantly higher in 
premenopausal acromegalic patients than in the normal population, and positively correlates with IGF1 levels and disease duration. Since increased mammographic breast density is an independent risk factor for cancer, these patients should be more carefully monitored (1).

Moreover, considering that mammography in dense breasts has a reduced diagnostic accuracy in comparison with the combined use of mammography and ultrasonography $(1,28)$, we suggest evaluating breasts of acromegalic patients as increased risk groups of patients.

\section{Declaration of interest}

The authors declare that there is no conflict of interest that could be perceived as prejudicing the impartiality of the research reported

\section{Funding}

This research did not receive any specific grant from any funding agency in the public, commercial or not-for-profit sector.

\section{References}

1 Boyd NF, Guo H, Martin LJ, Sun L, Stone J, Fishell E, Jong RA, Hislop G, Chiarelli A, Minkin S \& Yaffe MJ. Mammographic density and the risk and detection of breast cancer. New England Journal of Medicine 2007356 227-236. (doi:10.1056/NEJMoa 062790)

2 Byrne C, Schairer C, Wolfe J, Parekh N, Salane M, Brinton LA, Hoover R \& Haile R. Mammographic features and breast cancer risk: effects with time, age, and menopause status. Journal of the National Cancer Institute 199587 1622-1629. (doi:10.1093/jnci/ 87.21.1622)

3 Boyd NF, Byng JW, Jong RA, Fishell EK, Little LE, Miller AB, Lockwood GA, Tritchler DL \& Yaffe MJ. Quantitative classification of mammographic densities and breast cancer risk: results from the Canadian National Breast Screening Study. Journal of the National Cancer Institute 199587 670-675. (doi:10.1093/jnci/ 87.9.670)

4 Boyd NF, Stone J, Martin LJ, Jong R, Fishell E, Yaffe M, Hammond G \& Minkin S. The association of breast mitogens with mammographic densities. British Journal of Cancer $200287876-882$. (doi:10.1038/sj.bjc.6600537)

5 Byrne C, Colditz GA, Willett WC, Speizer FE, Pollak M \& Hankinson SE. Plasma insulin-like growth factor (IGF) I, IGFbinding protein 3, and mammographic density. Cancer Research $2000603744-3748$.

6 Maskarinec G, Williams AE \& Kaaks R. A cross-sectional investigation of breast density and insulin-like growth factor I. International Journal of Cancer 2003107 991-996. (doi:10.1002/ ijc.11505)

7 Lai JH, Vesprini D, Zhang W, Yaffe MJ, Pollak M \& Narod SA. A polymorphic locus in the promoter region of the IGFBP 3 gene is related to mammographic breast density. Cancer Epidemiology. Biomarkers and Prevention 200413 573-582.

8 Loeper S \& Ezzat S. Acromegaly: re-thinking the cancer risk. Reviews in Endocrine and Metabolic Disorders 20089 41-58. (doi:10.1007/s11154-007-9063-z)

9 Johansson H, Gandini S, Bonanni B, Mariette F, GuerrieriGonzaga A, Serrano D, Cassano E, Ramazzotto F, Baglietto L, Sandri MT \& Decensi A. Relationships between circulating hormone levels, mammographic percent density and breast cancer risk factors in postmenopausal women. Breast Cancer Research and Treatment $2008 \mathbf{1 0 8} 57-67$. (doi:10.1007/s10549007-9577-9)

10 Giustina A, Chanson P, Bronstein MD, Klibanski A, Lamberts S, Casanueva FF, Trainer P, Ghigo E, Ho K, Melmed S \& Acromegaly Consensus Group . A consensus on criteria for cure of acromegaly. Journal of Clinical Endocrinology and Metabolism 201095 3141-3148. (doi:10.1210/jc.2009-2670)

11 London SJ, Colditz GA, Stampfer MJ, Willett WC, Rosner B \& Speizer FE. Prospective study of relative weight, height, and risk of breast cancer. Journal of the American Medical Association 1989 262 2853-2858. (doi:10.1001/jama.262.20.2853)

12 Tagliafico A, Tagliafico G, Tosto S, Chiesa F, Martinoli C, Derchi LE \& Calabrese M. Mammographic density estimation: comparison among BI-RADS categories, a semi-automated software and a fully automated one. Breast 200818 35-40. (doi:10.1016/j.breast. 2008.09.005)

13 American College of Radiology (ACR). Breast Imaging Reporting And Data System (BI-RADS). 3rd edn. Reston, VA: American College of Radiology, 1998.

14 Tagliafico A, Resmini E, Nizzo R, Bianchi F, Minuto F, Ferone D \& Martinoli C. Ultrasound measurement of median and ulnar nerve cross-sectional area in acromegaly. Journal of Clinical Endocrinology and Metabolism 200893 905-909. (doi:10.1210/ jc.2007-1719)

15 Colao A, Ferone D, Marzullo P \& Lombardi G. Systemic complications of acromegaly: epidemiology, pathogenesis and management. Endocrine Reviews 200425 102-152. (doi:10. 1210/er.2002-0022)

16 Jenkins PJ. Cancers associated with acromegaly. Neuroendocrinology 200683 218-223. (doi:10.1159/000095531)

17 Bremnes Y, Ursin G, Bjurstam N, Rinaldi S, Kaaks R \& Gram IT. Insulin-like growth factor and mammographic density in postmenopausal Norwegian women. Cancer Epidemiology, Biomarkers \& Prevention 200716 57-61. (doi:10.1158/10559965.EPI-06-0788)

18 Boyd NF, Lockwood GA, Martin LJ, Knight JA, Byng JW, Yaffe MJ \& Tritchler DL. Mammographic densities and breast cancer risk. Breast Disease 199810 113-126.

19 Aiello EJ, Tworoger SS, Yasui Y, Stanczyk FZ, Potter J, Ulrich CM, Irwin M \& McTiernan A. Associations among circulating sex hormones, insulin-like growth factor, lipids, and mammographic density in postmenopausal women. Cancer Epidemiology, Biomarkers \& Prevention 200514 1411-1417. (doi:10.1158/ 1055-9965.EPI-04-0920)

20 Jenkins P, Mukherjeet A \& Shalet SM. Does growth hormone cause cancer? Clinical Endocrinology 200664 121. (doi:10.1111/j. 1365-2265.2005.02404.x)

21 Gullu BE, Celik O, Gazioglu N \& Kadioglu P. Thyroid cancer is the most common cancer associated with acromegaly. Pituitary 2010 13 242-248. (doi:10.1007/s11102-010-0224-9)

22 Nabarro JD. Acromegaly. Clinical Endocrinology $1987 \mathbf{2 6}$ 481-512. (doi:10.1111/j.1365-2265.1987.tb00805.x)

23 Hankinson SE, Willett WC, Colditz GA, Hunter DJ, Michaud DS, Deroo B, Rosner B, Speizer FE \& Pollak M. Circulating concentrations of insulin-like growth factor-I and risk of breast cancer. Lancet 1998351 1393-1396. (doi:10.1016/S01406736(97)10384-1)

24 Li BD, Khosravi MJ, Berkel HJ, Diamandi A, Dayton MA, Smith M \& $\mathrm{Yu}$ H. Free insulin-like growth factor-I and breast cancer risk. International Journal of Cancer 200191 736-739. (doi:10.1002/ 1097-0215(200002)9999:9999<::AID-IJC1111> 3.0.CO;2-)

25 Colao A, Balzano A, Ferone D, Panza N, Grande G, Marzullo P, Bove A, Iodice G, Merola B \& Lombardi G. Increased prevalence of colonic polyps and altered lymphocyte subset pattern in the colonic lamina propria in acromegaly. Clinical Endocrinology 1997 47 23-28. (doi:10.1046/j.1365-2265.1997.00253.x)

26 Colao A, Pivonello R, Auriemma RS, Galdiero M, Ferone D, Minuto F, Marzullo P \& Lombardi G. The association of fasting insulin concentrations and colonic neoplasms in acromegaly: a 
colonoscopy-based study in 210 patients. Journal of Clinical Endocrinology and Metabolism 200792 3854-3860. (doi:10. 1210/jc.2006-2551)

27 Wasif N, Garreau J, Terando A, Kirsch D, Mund DF \& Giuliano AE. MRI versus ultrasonography and mammography for preoperative assessment of breast cancer. American Surgeon 200975 970-975.

28 Sardanelli F, Boetes C, Borisch B, Decker T, Federico M, Gilbert FJ, Helbich T, Heywang-Köbrunner SH, Kaiser WA, Kerin MJ, Mansel RE, Marotti L, Martincich L, Mauriac L, Meijers-Heijboer H, Orecchia R,
Panizza P, Ponti A, Purushotham AD, Regitnig P, Del Turco MR, Thibault F \& Wilson R. Magnetic resonance imaging of the breast: recommendations from the EUSOMA working group. European Journal of Cancer 201046 1296-1316. (doi:10.1016/j.ejca.2010. 02.015)

Received 22 October 2010

Accepted 13 December 2010 